\title{
ENFORCEMENT OF CONSTRAINTS AND MAXIMUM PRINCIPLES IN THE VARIATIONAL MULTISCALE METHOD
}

\author{
JOHN A. EVANS, THOMAS J.R. HUGHES, AND GIANCARLO SANGALLI
}

\begin{abstract}
We derive an explicit formula for the fine-scale Greens function arising in variational multiscale analysis. The formula is expressed in terms of the classical Greens function and a projector which defines the decomposition of the solution into coarse and fine scales. The theory is presented in an abstract operator format and subsequently specialized for the advection-diusion equation. It is shown that different projectors lead to fine-scale Greens functions with very different properties. For example, in the advection-dominated case, the projector induced by the $H_{0}^{1}$-seminorm produces a fine-scale Greens function which is highly attenuated and localized. These are very desirable properties in a multiscale method, and ones that are not shared by the $L^{2}$-projector. By design, the coarse-scale solution attains optimality in the norm associated with the projector. This property, combined with a localized fine-scale Greens function, indicates the possibility of effective methods with local character for dominantly hyperbolic problems.

We also present a theoretical framework for the enforcement of inequality constraints. This approach borrows heavily from results in constrained and convex optimization.

The methodology described enables the development of numerical methods which satisfy predefined attributes. A practical and effective procedure for solving the steady advectiondiffusion equation is presented based on a VMS-inspired stabilized method, weakly enforced Dirichlet boundary conditions, and enforcement of a maximum principle and conservation constraint.
\end{abstract}

Keywords: variational multiscale analysis, constrained optimization, convex optimization, Lagrange multipliers, projection, fine-scale Green's function, advection-di ffusion, maximum principles, non-negativity, conservation, weak boundary conditions

\section{REFERENCES}

[1] J.A. Evans, T.J.R. Hughes, G. Sangalli, Enforcement of Constraints and Maximum Principles in the Variational Multiscale Method, Computer Methods in Applied Mechanics and Engineering, Vol. 199, pp. 61-76, 2009.

[2] T.J.R. Hughes, G. Sangalli, Variational Multiscale Analysis: the Fine-scale Green's Function, Projection, Optimization, Localization, and Stabilized Methods, SIAM J. Numer. Anal., Vol. 45 (2), pp. 539-557, 2007

The University of Texas at Austin Institute for Computational Engineering and Sciences 1 University Station C0200 Austin, TX 78712-0027, U.S.A

The University of Texas at Austin Institute for Computational Engineering and Sciences 1 University Station C0200 Austin, TX 78712-0027, U.S.A

Dipartimento di Matematica,Università di Pavia; Via Ferrata, 1; 27100 Pavia; Italy.

E-mail address: giancarlo.sangalli@unipv.it 